\title{
Satisfacción laboral en pediatras del conurbano bonaerense, Región Sur
}

\author{
MARIANA MARUCCO ${ }^{1}$, PEDRO R. GIL-MONTE ${ }^{2}$ \\ 1. Pediatra. Doctora en Salud Mental Comunitaria. Servicio de Pediatría. HZGA. Dr. A. Oñativia. Bs. As. (Argentina). \\ 2. Profesor Titular de Psicología del Trabajo y de las Organizaciones. Doctor en Psicología del Trabajo y de las Organizaciones. \\ Unidad de Investigación Psicosocial de la Conducta Organizacional (UNIPSICO) (Universidad de Valencia). \\ Facultad de Psicología (Departamento Social).
}

\begin{abstract}
Job Satisfaction in Pediatricians from the southern area of Buenos Aires

Objective: To determine the prevalence of job satisfaction in a sample of pediatricians. Method: 123 pediatricians, 89 women $(72,4 \%)$, working in Pediatrics Units of general hospitals. The study was crosssectional and non-randomized. Job satisfaction was evaluated by a scale of 8 items obtained from the "Job Satisfaction Questionnaire S20/23"1. Results: 13\% of cases manifested to be "dissatisfied" or "very dissatisfied" in the results of the scale. More than $50 \%$ of the participants manifested to be "very dissatisfied" or "dissatisfied" with the salary $(89,4 \%)$, hygiene of the working place $(57,7 \%)$, formation $(56,9 \%)$ and promotion possibilities $(59,3 \%)$. Significant differences were not obtained according to gender. Conclusions: Labor conditions for pediatricians need to change by means of public investments, in order to improve the quality of service offered to society.

(Key words: job satisfaction, pediatricians, quality of working life, attitudes).

Rev Chil Pediatr 2007; 78 (5): 489-493
\end{abstract}

\section{RESUMEN}

Objetivo: El objetivo de este estudio fue determinar la prevalencia de satisfacción laboral en una muestra de pediatras. Pacientes y Método: La muestra la formaron 123 pediatras que trabajaban en Servicios de Pediatría de hospitales generales, 89 mujeres $(72,4 \%)$ y 34 hombres $(27,6 \%)$. El estudio fue transversal, no aleatorizado. La satisfacción laboral se evaluó mediante una escala de 8 ítems del Cuestionario de Satisfacción laboral S20/23 ${ }^{1}$. Resultados: El 13\% de los participantes manifestaron estar insatisfechos o muy insatisfechos con los aspectos evaluados de su trabajo. El 89,4\% de los participantes manifestaron estar muy insatisfechos o insatisfechos con el salario $(89,4 \%)$, la higiene del lugar de trabajo $(57,7 \%)$, la formación $(56,9 \%)$, y con las posibilidades de promoción $(59,3 \%)$. No se obtuvieron diferencias significa-

Trabajo recibido el 15 de enero de 2007, devuelto para corregir el 16 de mayo de 2007, segunda versión el 8 de junio de 2007, aceptado para publicación el 10 de septiembre de 2007.

Correspondencia a:

Mariana Marucco

E-mail: marianamarucco@speedy.com.ar
Pedro R. Gil-Monte

E-mail: Pedro.Gil-Monte@uv.es 
tivas en función del sexo. Conclusiones: Los resultados evidencian el alto grado de insatisfacción en importantes aspectos de la vida laboral de los pediatras de hospitales generales. Se proponen recomendaciones para mejorar las variables evaluadas.

(Palabras clave: satisfacción laboral, pediatras, calidad de vida laboral, actitudes).

Rev Chil Pediatr 2007; 78 (5): 489-493

\section{Introducción}

La satisfacción laboral es uno de los indicadores más clásicos y a los que con mayor frecuencia se recurre cuando se persigue conocer cuál es la actitud general de las personas hacia su vida laboral. Esto es así debido a que el grado de satisfacción puede afectar la cantidad y calidad del trabajo que desempeñan los sujetos, además de incidir sobre otros aspectos como la baja calidad de la prestación, el ausentismo laboral, la propensión a abandonar la organización, etc ${ }^{2}$.

Desde la perspectiva de la Psicología de las Organizaciones la satisfacción laboral se puede definir como un estado emocional positivo o placentero resultante de la percepción subjetiva de las experiencias laborales del sujeto ${ }^{3}$. La satisfacción laboral se puede interpretar como que los profesionales tienen alguna idea más o menos definida de cómo creen que deberían de ser las cosas en su trabajo y los aspectos importantes relacionados con él. Depende de las relaciones sociales que mantienen los profesionales dentro de la organización, de sus características personales, de sus valores y de las expectativas que tienen acerca del trabajo. Los profesionales saben que les gustaría y como les gustaría que fuesen las cosas. Estas ideas son comparadas con la realidad, y de esa comparación surge un juicio y una actitud asociada: las personas están más o menos satisfechas ${ }^{2}$.

La satisfacción laboral se ha considerado una consecuencia actitudinal al estrés laboral. Es uno de los indicadores más clásicos para conocer la actitud general de las personas hacia su vida laboral. Esto es así debido a que el grado de satisfacción puede afectar a la cantidad y calidad del trabajo que desempeñan los profesionales. La satisfacción laboral en la atención sanitaria se ha igualado en importancia a la preparación científica del profesional o a la disposición de una determinada tecnología, y es indiscutible que al determinar la satisfacción laboral se está midiendo la calidad de la organización y de sus servicios internos ${ }^{4}$. Los indicadores de satisfacción laboral incluyen: satisfacción intrínseca con el trabajo, satisfacción con las condiciones de trabajo, satisfacción con las prestaciones recibidas, satisfacción con la supervisión, satisfacción con la participación, y otros factores de la satisfacción labo$\mathrm{ral}^{2}$.

Existen pocos estudios sobre satisfacción laboral en pediatras que nos informen sobre niveles de prevalencia en este colectivo profesional, y cómo afecta a su calidad de vida laboral y a la calidad del servicio que ofrecen a la sociedad. Jiménez, Morales y Martínez ${ }^{5}$, en un estudio realizado con cirujanos pediatras, concluyen que los niveles de satisfacción eran bajos o medios, y con una relación positiva y significativa con el nivel jerárquico, siendo la satisfacción intrínseca uno de los principales indicadores que promovieron la satisfacción laboral. En muestras menos especializadas, Velarde-Ayala, Priego-Álvarez, López-Naranjo ${ }^{6}$, concluyen en un estudio realizado con médicos que existe insatisfacción laboral, que hay problemas con la gerencia dado que se carece de procedimientos orientados al capital humano, existe desorganización, desequilibrio entre la oferta y la demanda, y un sueldo insuficiente que contribuye al fenómeno del poliempleo médico. López-Soriano, Bernal y Canovas ${ }^{7}$, informan que la satisfacción global de los profesionales sanitarios fue de nivel medio.

El objetivo de este estudio es determinar la prevalencia de satisfacción laboral en una muestra de pediatras y analizar sus relaciones con algunas variables de carácter sociodemográfico. 


\section{Participantes y Método}

La muestra quedó conformada por 123 pediatras, 89 mujeres $(72,4 \%)$ y 34 hombres $(27,6 \%)$. La media de edad fue de 42,38 años (min. $=24, \max =70$ años).

El estudio fue transversal, y no aleatorizado. Fueron incluidos todos los pediatras con nombramiento de 36 horas semanales, con funciones de planta y guardia que trabajan en los Servicios de Pediatría de Hospitales Generales de la Región Sanitaria VI del conurbano bonaerense, en Argentina. El universo de pediatras rentados en esta Región Sanitaria es de 228. Ciento un participantes refirieron tener pareja estable $(82,10 \%)$ y 22 no tener pareja estable (17,9\%). Según tipo de contrato, 33 participantes $(26,8 \%)$ trabajan como personal de planta de 36 horas semanales (no realizaban guardia) y 90 participantes $(73,2 \%)$ realizan una guardia semanal de 24 horas más 12 horas de trabajo en consultorios externos o internación. La media de antiguedad en la profesión fue de 12,85 años $(d t=9,33)$.

La satisfacción laboral se evaluó mediante 8 ítem extraídos del "Cuestionario de satisfacción laboral S20/23" . Los items preguntan sobre el grado de satisfacción con diferentes aspectos del trabajo (intrínseca, salario, objetivos y metas, higiene, formación, promoción, supervisión, y participación en toma de decisiones). Se responden con una escala tipo Likert de cinco grados que va de 0 ("Muy insatisfecho) a 4 (Muy satisfecho) (alfa $=.75)$. Además se incluyeron algunas variables sociodemográficas (sexo, estado civil, tipo de contrato, antigüedad en la profesión). Fue anónimo y autoadministrado.

\section{Resultados}

La tasa de respuesta sobre los cuestionarios distribuidos se situó en un 53,94\%, pero no fue uniforme (del 15 al 100\%). La escala de satisfacción laboral obtuvo un valor de consistencia interna alfa de Cronbach adecuado ${ }^{8}$. También resultaron adecuados los valores de asimetría $=$ -29 , de curtosis $=-22$ y test Kolmogorov-Smirnov, $0,693, \mathrm{p}=0,723$ ".
No se obtuvieron relaciones significativas entre las variables sociodemográficas y la escala de satisfacción laboral.

Según los resultados sobre la prevalencia de satisfacción laboral en la muestra, el 13\% de los participantes manifestaron estar muy insatisfechos o insatisfechos, mientras que sólo un $3 \%$ se situaron en valores superiores a satisfecho. El resto de sujetos se ubicaron en un rango de indiferencia, con valores medios superiores a insatisfecho, pero inferiores a satisfecho (tabla 1).

Para los diferentes indicadores de satisfacción se obtuvieron los siguientes resultados (figura 1). Para la satisfacción con las oportunidades que le ofrece su trabajo de hacer las cosas que le gustan (satisfacción intrínseca), el 4,1\% manifestó estar muy insatisfecho y el $23,6 \%$ insatisfecho, frente a un $50,4 \%$ que se mostraban satisfechos, y un $11,4 \%$ muy satisfechos. Respecto a la satisfacción con el salario, el 47,2\% estaban muy insatisfechos, y el $42,3 \%$ insatisfechos. La evaluación de los $o b-$ jetivos, metas y tasas de producción que se deben alcanzar, presentó que frente al 34,1\% que se manifestaban insatisfechos, y al 32,5\% que se manifestaban satisfechos, el $21,1 \%$ se manifestaron indiferentes. La limpieza e higiene del lugar de trabajo también resultó un indicador de insatisfacción laboral, pues el 43,9\% de los participantes se mostraron insatisfechos con este indicador, y un 13,8\% muy insatisfechos. Un porcentaje similar se obtuvo para la

Tabla 1. Descriptivos para la escala de satisfacción laboral y sus indicadores

\begin{tabular}{lrrc}
\hline & Media & dt & Moda \\
\hline Satisfacción laboral (escala) & 1,81 &, 62 & 2 \\
1. Hacer cosas que gustan & 2,41 & 1,09 & 3 \\
2. Salario &, 71 &, 85 & 0 \\
3. Objetivos y metas & 1,87 & 1,07 & 1 \\
4. Limpieza e higiene & 1,65 & 1,17 & 1 \\
5. Formación & 1,63 & 1,14 & 1 \\
6. Promoción & 1,46 & 1,03 & 1 \\
7. Supervisión & 2,37 &, 91 & 3 \\
8. Participación & 2,37 & 1,01 & 3 \\
\hline
\end{tabular}




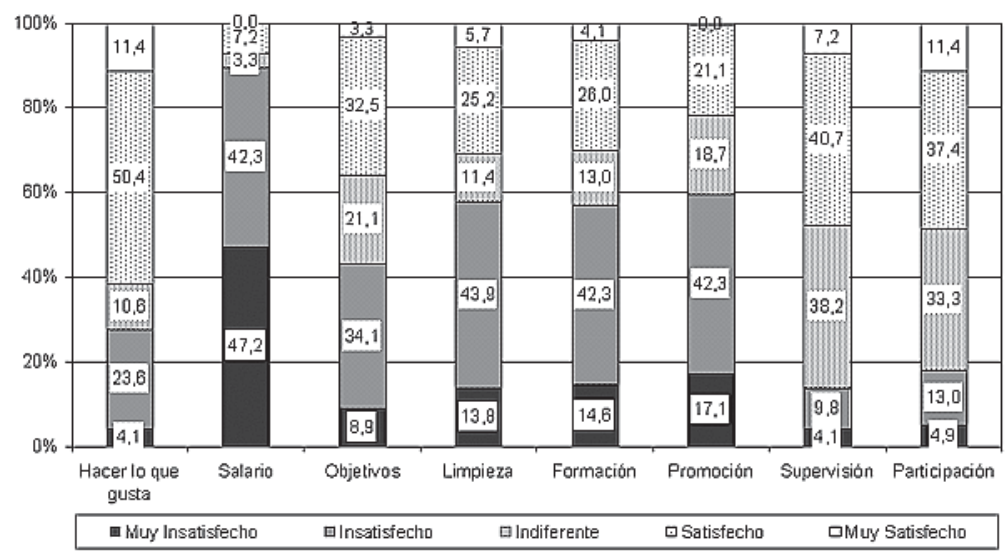

Figura 1. Porcentaje de sujetos por niveles en las indicadores de satisfacción laboral.

satisfacción con las oportunidades de formación (42,3\% manifestaron insatisfacción, y $14,6 \%$ mucha insatisfacción), y con las oportunidades de promoción (42,3\% manifestaron insatisfacción, y 17,1\% mucha insatisfacción). Los niveles de satisfacción con la supervisión resultaron más elevados, pues un $40,7 \%$ de los participantes expresó estar satisfecho con la supervisión. Si bien, un 38,2\% expresó indiferencia hacia este indicador. Por último, un resultado similar se obtuvo para la satisfacción con la participación, donde un $37,4 \%$ de los participantes se manifestó satisfecho, y un 33,3\% indiferente.

\section{Discusión}

La tasa de respuesta obtenida es similar y superior a otros estudios realizados en pediatras $^{5}$, por lo que los resultados son útiles para el objetivo perseguido con este estudio. No obstante, entre las debilidades del estudio, hay que tener presente que no se valoran todos los indicadores de satisfacción, y que el muestreo no fue aleatorio lo que puede afectar a la validez externa de los resultados. Existen otros factores no analizados en este estudio (abordaje multicausal) que podrían influir en la satisfacción laboral: el contexto de la sanidad pública en la provincia de Buenos aires, la burocratización, la falta de personal de apoyo en las consultas, el escaso tiempo de consulta, el desequilibrio entre las expectativas del paciente y del pediatra, etc. También hay que tener presente que el muestreo no fue aleatorio, por lo que los niveles de satisfacción pueden ser más bajo debido a que los individuos que no responden en estudios de este tipo suelen presentar niveles de satisfacción inferiores a los que sí contestan.

En general, los pediatras del estudio se muestran en gran medida insatisfechos con el salario $(89,5 \%)$, con los objetivos y metas que deben alcanzar $(43,1 \%)$, con las condiciones higiénicas de su lugar de trabajo $(57,7 \%)$, con las oportunidades de formación $(56,9 \%)$, y con las oportunidades de promoción (59,3\%). Por el contrario, se manifiestan satisfechos con las oportunidades de hacer las cosas que les gus$\tan (61,8 \%)$, con la supervisión recibida (48\%), y con su participación en las decisiones del servicio $(48,8 \%)$. Llama la atención, el porcentaje de pediatras con indiferencia frente a los 
objetivos, metas y tasas de producción $(21,1 \%)$, la supervisión $(38,2 \%)$, y la participación en la toma de decisiones del servicio (33,3\%).

Los niveles de satisfacción han resultado inferiores a los obtenidos para pediatras españoles. En nuestro estudio la media fue de 1,81 (rango 0-4), mientras que en el estudio de Jiménez et $\mathrm{al}^{5}$, fue de 3,43 (rango 1-5).

Algunas recomendaciones pueden derivarse de los resultados del estudio. En primer lugar, los niveles de satisfacción obtenidos llevan a concluir que se deben llevar a cabo acciones para mejorar la satisfacción laboral de los pediatras del conurbano bonaerense con el fin de mejorar su calidad de vida laboral y el servicio que ofrecen a la comunidad. Las acciones deben ir, al menos, dirigidas a dos niveles. En el nivel social se hace necesario realizar inversiones en el sistema nacional de salud con el fin de mejorar el nivel salarial de los pediatras. Esta variable es un factor de higiene o de satisfacción extrínseca9. También se hace necesario mejorar las condiciones físicas del lugar de trabajo mediante inversiones en infraestructuras hospitalarias.

Paralelamente, se recomiendan intervenciones a nivel organizacional. Los altos índices de satisfacción con las oportunidades que frece el trabajo de hacer las cosas que gustan es una oportunidad para que los gestores y jefes de servicio potencien la satisfacción. En este sentido programas de desarrollo organizacional ${ }^{10}$, en los que se potencie la participación de los trabajadores, pueden contribuir a mejorar la satisfacción con los objetivos y metas que deben alcanzar y con las oportunidades de formación, al tiempo que pueden contribuir a dismi- nuir los niveles de indiferencia con la supervisión recibida. También es recomendable desarrollar programas de entrenamiento en habilidades de dirección y liderazgo transformacional ${ }^{11}$ a los jefes de servicio.

\section{Referencias}

1.- Meliá JL, Peiró JM: La medida de la satisfacción laboral en contextos organizacionales: el cuestionario de satisfacción S20/23. Psicologemas 1989; 3: 59-74.

2.- Peiró JM, Luque O, Meliá JL, Los Certales F: El estrés de enseñar. Sevilla: Alfar; 1981.

3.- Locke EL: The nature and causes of job satisfaction. En: M. D. Dunnette (Ed.), Handbook of Organizational and Industrial Psychology. Chicago: Rand Mc Nally 1976; 1297-349.

4.- Fernández MI, Moinelo A, Villanueva A, et al: Satisfacción laboral de los profesionales de Atención Primaria del área 10 del Insalud de Madrid. Rev Esp Salud Pública 2000; 74: 139-47.

5.- Jiménez C, Morales JL, Martínez C: Estudio del síndrome de "Burnout" en cirujanos pediatras españoles. Cir Pediatr 2002; 15: 73-8.

6.- Velarde-Ayala RJ, Priego-Álvarez, López-Naranjo JI: Estudio de satisfacción laboral del médico de la consulta externa de medicina familiar del centro médico ISSET. Hit Cien Econ Admin 2004; 27: 55-66.

7.- López-Soriano F, Bernal L, Canovas A: Satisfacción laboral de los profesionales en un Hospital Comarcal de Murcia. Rev Cal Asisten 2001; 16: 243-2.

8.- Nunnaly NC: Psychometric theory. Nueva York: McGraw-Hill; 1978.

9.- Herzberg F, Mausner B, Snyderman BB: The motivation to work ( $2^{\mathrm{a}}$ ed.). Nueva York: John Wiley \& Sons; 1959.

10.- French WL, Bell CH Jr: Desarrollo organizacional. México: Prentice-Hall; 1996.

11.- Barling J, Weber T, Kelloway EK: Effects of transformational leadership training on attitudinal and financial outcome: A field experiment. J Appl Psychol 1996; 81: 827-32. 